\section{Rethinking the way we approach eating disorders}

Jones et al (2012) detail the clinical features of eating disorders, and emphasise the role of the general psychiatrist in their detection and management. Despite the increased interest of both psychiatry and the media in eating disorders over the past two decades, this appears to remain a neglected clinical area. The mainstay of treatment is largely psychological, a skills set not always well developed among psychiatrists. Junior trainee experience in this area is often limited, with specialist services not routinely offering placements.

Jones et al give a very good summary of the core and more general features of both anorexia nervosa and bulimia nervosa, but only briefly mention 'eating disorders not otherwise specified' (EDNOS), which include binge eating disorder. Although there tends to be more focus on anorexia and bulimia, it is increasingly recognised that a large proportion of eating disorders do not fit into these two categories, with EDNOS still the most common diagnosis. Importantly, Crow et al (2009) found increased mortality rates among people with EDNOS similar to those found among those with anorexia nervosa. It is also not uncommon for people to move between eating disorder diagnoses, with diagnostic cross-over from anorexia or bulimia to EDNOS in over a third of cases (Milos 2005).

It may therefore be helpful to think about eating disorders as dimensional or on a spectrum, rather than as specific categories. The transdiagnostic approach proposed by Fairburn and colleagues, and adopted by some specialist eating disorder services, is based on the recognition that most eating disorders share certain core features. These include an extreme concern about eating, weight and body shape and the ability to control them, over-evaluation of the self on the basis of weight and shape, and engagement in weight control behaviours as a consequence (Fairburn 2005). This is a potentially useful and pragmatic approach to the treatment of eating disorders that requires further exploration.

Fairburn CG, Bohn K (2005) Eating disorders NOS (EDNOS): an example of troublesome 'not otherwise specified' (NOS) category in DSM-IV. Behaviour Research and Therapy 43: 691-701.

Jones WR, Schelhase M, Morgan JF (2012) Eating disorders: clinical features and the role of the general psychiatrist. Advances in Psychiatric Treatment 18: 34-43.

Milos G, Spindler A, Schnyder U, et al (2005) Instability of eating disorder diagnoses: prospective study. British Journal of Psychiatry 187: 573-8.
Crow CJ, Peterson CB, Swanson SA, et al (2009) Increased mortality in bulimia nervosa and other eating disorders. American Journal of Psychiatry 166: 1342-6.

Marlene M. Kelbrick, ST4, Leicestershire Partnership NHS Trust. Email: Marlene.Kelbrick@nhft.nhs.uk

doi: 10.1192/apt.18.4.315

\section{Dementia classification}

Gupta and colleagues' description of rarer forms of dementia, which draws attention to estimates of prevalence rates, is helpful for clinicians (Gupta 2012). As there are more than 75 diseases that cause signs and symptoms of dementia, the differential diagnosis can be extensive. A useful way to classify dementias is into one of three categories: reversible, non-progressive or progressive (Rabins 2008).

A number of potentially reversible causes of dementia have been identified. The most common are medication-induced cognitive decline, hypothyroidism, major depression, normal-pressure hydrocephalus and alcoholism. These reversible causes account for around $1-2 \%$ of cases presenting for evaluation. However, many potentially reversible causes do not improve after treatment. Non-progressive dementias include those that follow head trauma and stroke, while progressive dementias include those with common causes (for example, Alzheimer's disease) and those with rarer causes (for example, Creutzfeldt-Jakob disease).

Another classifier, pseudodementia, used to be used to refer to the dementia syndrome sometimes seen in major depression (Rabins 2008). It was thought to be 'pseudo' because it was reversible, and at the time the definition of dementia included irreversibility. This criterion has since been removed from the major classification systems. The dementia of depression is a 'real' dementia in that it meets the current defining characteristics of the syndrome.

In an article on late-onset depressive disorder (Yadav 2010), I draw clinicians' attention to this fact and advise them to be wary. Although the cognitive impairment in major depressive disorder may initially be 'pseudodementia', i.e. reversible, in some cases it progresses to a true, i.e. irreversible, dementia. Aggressive treatment of the depression and timely referral, investigation and treatment of cognitive impairment in late-onset depressive disorder are of paramount importance (Alexopoulos 1993). 
Alexopoulos GS, Meyers BS, Young RC, et al (1993) The course of geriatric depression with 'reversible dementia': a controlled study. American Journal of Psychiatry 150: 1693-9.

Gupta S, Fiertag 0, Thanulingam T, et al (2012) Further rare and unusual dementias. Advances in Psychiatric Treatment 18: 67-77.

Rabins PV, Slavney PR (2008) Overview of psychiatric symptoms and syndromes. In Psychiatric Aspects of Neurologic Diseases: Practical Approaches to Patient Care (eds CG Lyketsos, PV Rabins, JR Lipsey, et al): 41-64. Oxford University Press.

Yadav DS (2010) Late onset depressive disorder. Geriatric Medicine 40: $34-7$

Devender Singh Yadav, Specialty Doctor in Psychiatry, Abertawe Bro Morgannwg University Local Health Board, Wales. Email: dsyadav@doctors.org.uk

doi: 10.1192/apt.18.4.315a

\section{Multiple diagnostic entities model may better fit schizoaffective disorder to nosology}

In his brief 'refreshment', Castle (2012) considers the very topical subject of 'schizoaffective disorder'. Because of their low reliability and questionable validity, there is need for a revision of the current diagnostic concepts of schizoaffective disorder, but developments in ICD-11 and DSM-5 are as yet unclear. How we might see this disorder in future nosology is an interesting area of discussion. Psychiatric nosology can be conceptualised in terms of three models: Kraepelin's dichotomous approach, the dimensional diagnostic approach and the multiple diagnostic approach (Fig. 1).

A dichotomous classification of non-organic psychoses is not compatible with recent neurobiological findings. Furthermore, various studies of psychopathological symptoms have also failed to confirm the dichotomous classification. Rather,

Dichotomous approach
Schizophrenia
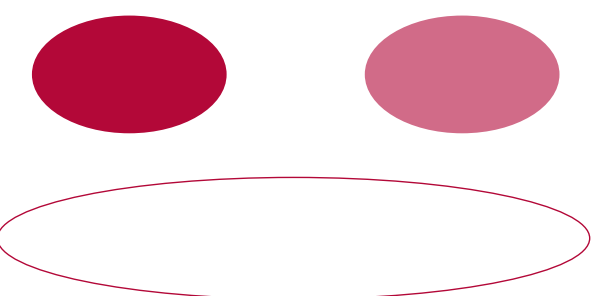

Multiple entities approach they point to a continuous-spectrum model of functional psychoses using a more dimensional diagnostic approach. However, at this point, switching from a categorical classification to a purely dimensional model entails hazards in clinical practice. This is because current treatment guidelines are based on categorical diagnoses, and the psychopathological syndrome dimensions have low stability over the long-term course of the illness. An alternative would be subdivision of the classic categories into multiple diagnostic entities. In such a model, distinct aetiological factors, psychopathological characteristics, neuropathological findings and outcomes can be entertained simultaneously.

Further, one can classify non-organic psychoses on the basis of course and outcome, which might improve the individual treatment of psychiatric disorders. Following Kasanin's original concept (Kasanin 1933), future diagnostic systems could try to establish reliable criteria for schizoaffective disorder that predict a favourable outcome and allow a differentiation from chronic schizophrenic disorders. However, it would be interesting to see the relationship of schizoaffective disorder to brief and acute psychoses. Some authors have argued against this approach because distinct categories for remitting psychoses such as schizoaffective disorders could contribute to increasing the stigma of schizophrenia.

The forthcoming ICD-11 and DSM-5 might follow a 'triaxial' classification similar to that proposed by Essen-Moller (1962). Jager et al (2008) speculated that they will introduce the complementary use of dimensional and categorical concepts. A dimensional concept can be helpful in describing the cross-sectional clinical picture, whereas a categorical approach can specify course and outcome. Psychopathological course types can be considered as prototypes within a continuous biological spectrum of schizophrenic and affective disorders. An additional 'axis' could comprise information about aetiology. Many authors (e.g. Lake 2007; Malhi 2008) propose the omission of the current concept of schizoaffective disorder from ICD-11 and DSM-5. Thus, a model of multiple diagnostic entities may be compatible with retaining the diagnostic category of schizoaffective disorder in the nosology.

Castle DJ (2012) Schizoaffective disorder. Advances in Psychiatric Treatment 18: 32-3.

Essen-Moller E (1962) On classification of mental disorders. Acta Psychiatrica Scandinavica 37: 119-26.

Jager M, Frasch K, Becker T (2008) New ways in psychiatric diagnostics? Fortschritte der Neurologie - Psychiatrie 76: 186-293. 